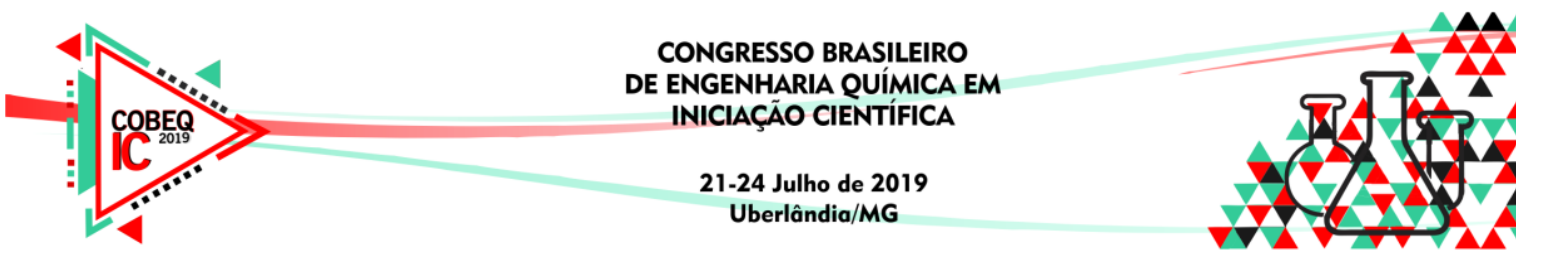

\title{
SIMULAÇÃO E AVALIAÇÃO EXERGÉTICA DO PROCESSO DE PRÉ-TRATAMENTO ÁCIDO DILUÍDO E HIDRÓLISE ENZIMÁTICA DA PALHA DO MILHO
}

\author{
A. B. R. JESUS ${ }^{1}$, P.C. ZANESCO ${ }^{1}$, A.C.O. MAFRA ${ }^{1}$, W. C. SILVA ${ }^{1}$ \\ ${ }^{1}$ Universidade Federal de Mato Grosso, Faculdade de Engenharia \\ E-mail para contato: rodrigues.oct@gmail.com
}

\begin{abstract}
RESUMO - O presente estudo avaliou o desempenho do processo de prétratamento ácido diluído e hidrólise enzimática da palha de milho para produção de etanol de $2^{\mathrm{a}}$ geração. Foram realizadas avaliações exergéticas das correntes e dos equipamentos presentes no processo. Para tal, desempenhou-se uma análise de sensibilidade das temperaturas de operação do reator de pré-tratamento em função da perda exergética. Os processos e a análise exergética foram simulados no software Aspen Plus ${ }^{\circledR}$ versão 8.6. Os resultados obtidos indicam que a perda de exergia total foi em média de $450 \mathrm{~kW}$. Dentre os equipamentos avaliados, constatou-se que o reator de pré-tratamento apresentou a maior perda exergética, motivado pela alta influência da temperatura.
\end{abstract}

\section{INTRODUÇÃO}

O milho é um vegetal que pode ser utilizado para a produção de etanol de $1^{a}$ geração, processo este que beneficia apenas os seus grãos. Em síntese, nessa rota de produção, alguns resíduos biomássicos são gerados, os quais são considerados de baixo valor agregado, o que levam a ser direcionados, em geral, à queima para geração de energia na indústria ou para aplicações pouco rentáveis. Sobretudo, estes insumos podem ser empregados para a produção de etanol, denominado de etanol de $2^{\mathrm{a}}$ geração ou etanol lignocelulósico, biocombustível que apresenta vantagem relevante em não competir com a produção de alimentos (Aditiya et al. 2016).

A produção de etanol lignocelulósico, em geral, apresenta três principais etapas: prétratamento, hidrólise-fermentação e o processo de separação. A etapa de pré-tratamento é considerada o gargalo para o desenvolvimento do processo, devido à alta dependência da sua eficiência para obter significativa conversões em substratos fermentativos na etapa de hidrólise. Em síntese, o consumo energético na etapa de pré-tratamento e hidrólise são determinantes para avaliar a eficiência energética do processo (Conde-Mejía et al. 2012).

Segundo Vučković et al. (2014), a exergia é estabelecida como o trabalho máximo quando a matéria é levada ao estado de equilíbrio termodinâmico com os componentes comuns de sua natureza circundante. A análise exergética é um método de alta aplicação para avaliação, ambiental, econômica e na otimização de processos de sistemas energéticos industriais, principalmente quando busca-se otimizar o consumo energético e reduzir emissões de efluentes ao meio (Szargut 1980). 


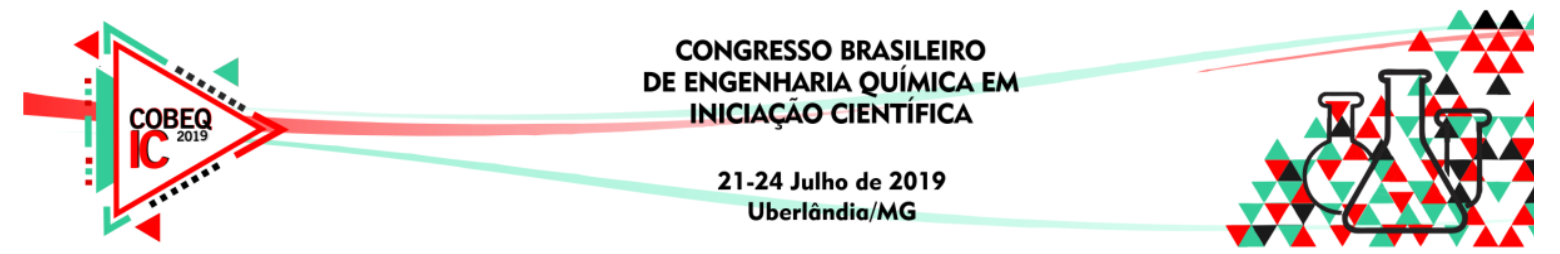

De acordo com Luyben (1996) a simulação computacional de processos é desempenhada, em geral, por ferramentas computacionais que são utilizadas na modelagem de plantas industriais e processamento de componentes químicos. Em suma, estes tipos de ferramentas permitem prever o comportamento do processo aplicando relações básicas de engenharia, de modo a contribuir nas identificações de parâmetros que influenciam o desenvolvimento e eficiência do processo abordado.

Nesse âmbito, dado os desafios que a produção de etanol de $2^{\mathrm{a}}$ geração apresenta, devido às incertezas dos potenciais impactos econômicos e ambientais do processo. O presente trabalho, teve como objetivo geral a simulação e avaliação exergética do processo de prétratamento e hidrólise da palha do milho para produção de etanol de $2^{\mathrm{a}}$ geração, com auxílio de ferramentas computacionais de engenharia.

\section{METODOLOGIA}

\subsection{Simulação do Processo}

O processo convencional de pré-tratamento e hidrólise da palha do milho é baseado no processo ácido diluído e hidrólise enzimática, no qual o ácido sulfúrico diluído e a enzima celulase atuam como substâncias determinantes na produção dos substratos do mosto que seguem para a etapa de fermentação alcóolica. O processo convencional de pré-tratamento e hidrólise foi simulado de acordo com Humbird et al. (2011). O processo de pré-tratamento ácido diluído e hidrólise enzimática da palha do milho estão descritos na Figura 1.

Figura 1: Fluxograma da etapa de pré-tratamento e hidrólise da palha de milho. Correntes principais: (BIOMASSA) componentes da palha de milho, (DIL-ACID) ácido sulfúrico diluído, (S2) vapor, (ENZIMA) enzima, (NH3) amônia, (MOST) mosto fermentativo e (S6) sólidos lignocelulósicos.

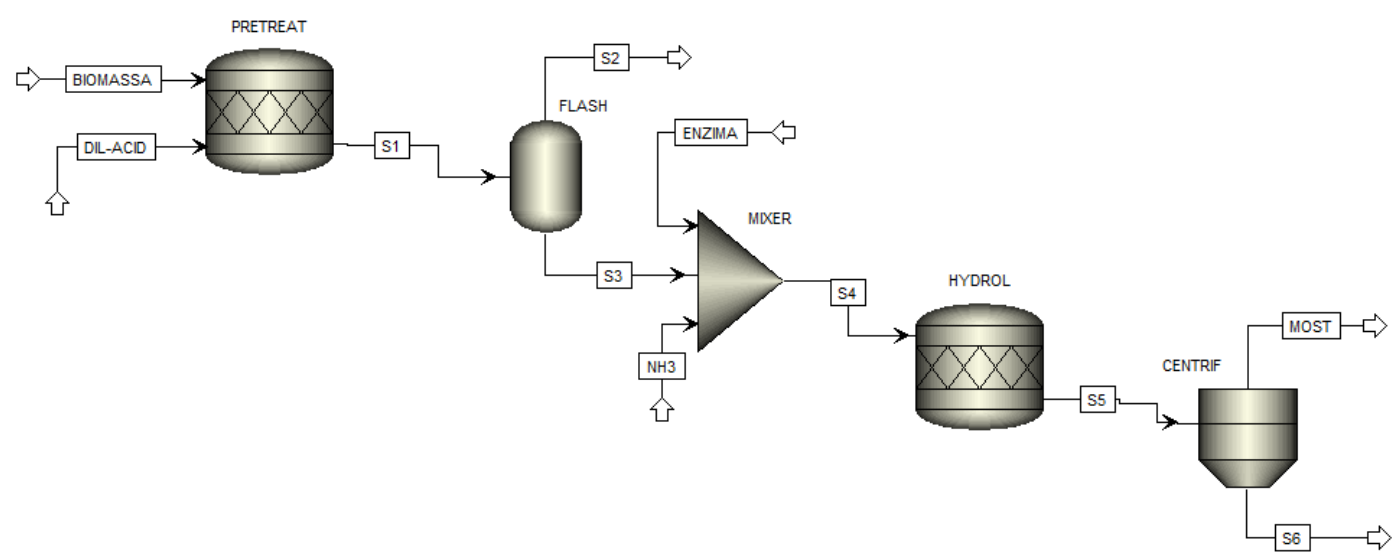

O programa computacional empregado na simulação do processo de pré-tratamento e hidrólise da palha do milho descrito na Figura 1 foi o Aspen Plus ${ }^{\circledR}$ v.8.6 (AspenTech, 2010). Os principais parâmetros dos reatores PRETREAT e HYDROL, empregados na simulação estão descritos na Tabela 1 e Tabela 2, respectivamente. 


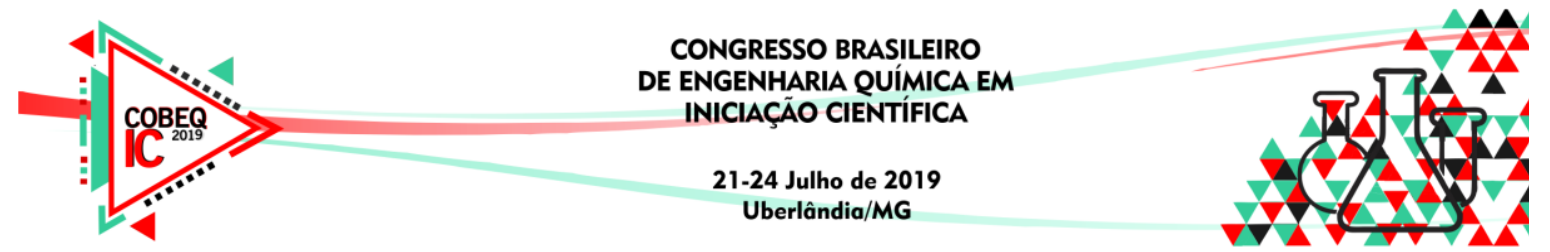

O conteúdo dos principais componentes da palha de milho (em base seca), de acordo com dados de Humbird et al. (2011), foi o seguinte: celulose, 37,4\% ; hemicelulose, 21,1\% ; lignina, $18 \%$ (p/p); proteínas, $3,10 \%$ e cinzas $5,23 \%$.

Tabela 1: Parâmetros e reações do reator de pré-tratamento

\begin{tabular}{cc}
\hline \multicolumn{2}{c}{ Reator de Pré-Tratamento } \\
\hline Carregamento de ácido sulfúrico & $18 \mathrm{mg} / \mathrm{g}$ biomassa seca \\
Temperatura & $158{ }^{\circ} \mathrm{C}$ \\
Pressão & $5,5 \mathrm{~atm}$ \\
\hline Reação & Conversão $(\%)$ \\
\hline Celulose $+\mathrm{H}_{2} \mathrm{O} \rightarrow$ Glicose & $9,9 \%$ \\
Xylan $+\mathrm{H}_{2} \mathrm{O} \rightarrow$ Xilose & $90,0 \%$ \\
Lignina $\rightarrow$ Lignina Solúvel & $5,0 \%$ \\
\hline
\end{tabular}

Fonte: Humbird et al. (2011).

O banco de dados das substâncias do processo, como enzimas e componentes biomássicos, foram baseados através das tabelas de propriedades físicas da NREL, elaborado pelos trabalhos de Wooley e Putsche (1996). Ademais, considerou-se apenas as reações predominantes para obter os substratos fermentativos (xilose e glicose) empregados para a produção de etanol.

Tabela 2: Parâmetros e reações do reator de hidrólise

\begin{tabular}{cc}
\hline \multicolumn{2}{c}{ Reator de Hidrólise } \\
\hline $\begin{array}{cc}\text { Carregamento de enzima } \\
\text { Temperatura }\end{array}$ & $20 \mathrm{mg}$ proteína/g celulose \\
Reação & $48{ }^{\circ} \mathrm{C}$ \\
\hline Celulose $+\mathrm{H}_{2} \mathrm{O} \rightarrow$ Glicose & $90,0 \%$
\end{tabular}

Fonte: Humbird et al. (2011).

O modelo termodinâmico NRTL (sigla em inglês para Non-random two-liquid model) foi empregado na simulação por ser o modelo que melhor ajusta as reações de equilíbrio.

\subsection{Análise Exergética}

De acordo com Ojeda et. al (2011) a análise exergética proporciona uma unificada e efetiva ferramenta para a avaliação da eficiência global de um processo, dado ao fato de ser uma análise baseada na Segunda Lei da Termodinâmica, com capacidade de localizar perdas termodinâmicas e identificar as origens e os impactos no ambiente natural.

Para um processo, em geral, no estado estacionário o balanço exergético de um fluxo pode ser expresso pela Equação 1:

$$
E=H-H_{0}-T_{0}\left(S-S_{0}\right)+\sum_{i} \mu_{i 0}\left(n_{i}-n_{i 0}\right)
$$




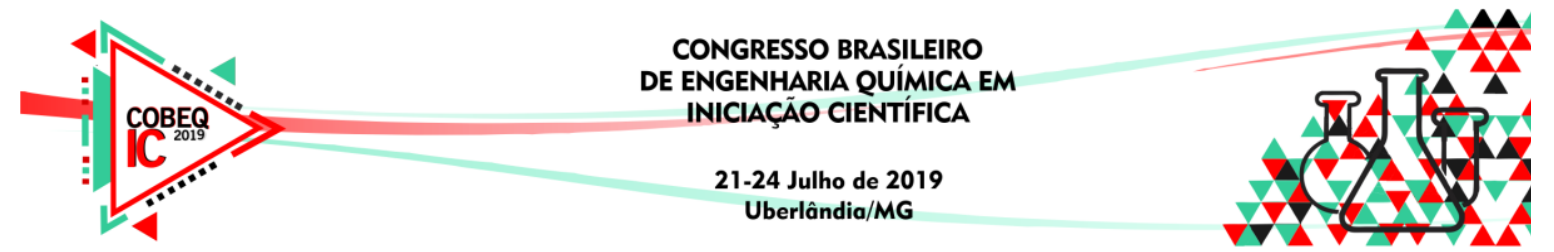

tal que, $E$ é dado como a exergia do fluxo, $H$ é a entalpia, $T_{0}$ é a temperatura inicial correspondente do fluxo, $S$ é a entropia e $\mu_{\mathrm{i} 0}$ é o número de mols da substância.

\section{RESULTADOS E DISCUSSÕES}

Os resultados das alimentações e saídas principais obtidas da simulação estudada são mostrados na Tabela 3. Na análise exergética, a taxa de exergia perdida foi calculada para cada corrente. A base de cálculo foi de 7943,30 kg/h de biomassa em solução. Fluxo mássico (kg/h), temperatura $\left(\mathrm{T},{ }^{\circ} \mathrm{C}\right)$, pressão $(\mathrm{P}, \mathrm{atm})$ e a exergia $(\mathrm{Ex}, \mathrm{kW})$ são apresentados para cada corrente.

Tabela 3: Parâmetros de entrada e saída da taxa mássica do processo

\begin{tabular}{lcccc}
\hline \multicolumn{1}{c}{ Corrente } & $\mathbf{F}(\mathbf{k g} / \mathbf{h})$ & $\mathbf{T}\left({ }^{\circ} \mathbf{C}\right)$ & $\mathbf{P}(\mathbf{a t m})$ & $\mathbf{E x}(\mathbf{k W})$ \\
\hline BIOMASS & 7943,30 & 158,00 & 5,50 & 496,74 \\
DIL-ACID & 198,10 & 50,00 & 5,50 & 1,24 \\
S1 & 8141,40 & 158,00 & 5,50 & 127,19 \\
S2 & 300,89 & 104,50 & 1,00 & 41,26 \\
S3 & 7840,51 & 104,50 & 1,00 & 40,38 \\
NH3 & 1513,61 & 36,00 & 1,00 & 4,70 \\
ENZIMA & 1316,20 & 29,00 & 1,00 & 1,66 \\
S4 & 24292,81 & 48,51 & 1,00 & 24,83 \\
S5 & 24292,81 & 48,00 & 1,00 & 13,62 \\
MOST & 22507,51 & 48,00 & 1,00 & 13,08 \\
S6 & 1785,30 & 48,00 & 1,00 & 0,55 \\
\hline
\end{tabular}

A conversão global dos compostos lignocelulósicos do processo de pré-tratamento ácido diluído seguido pelo processo de hidrólise enzimática foi de $91 \%$ em glicose e $90 \%$ em xilose.

O balanço exergético para cada equipamento foi obtido através do balanço das correntes do processo o qual, a porcentagem de perda total de exergia representa a taxa de trabalho destruído devido às irreversibilidades que estão inseridas no processo. A Tabela 4 mostra o balanço de exergia dos equipamentos do processo de pré-tratamento e hidrólise.

Tabela 4: Balanço de exergia dos equipamentos do processo de pré-tratamento e hidrólise

\begin{tabular}{ccccc}
\hline Equipamento & $\begin{array}{c}\text { Exergia da } \\
\text { Entrada }(\mathbf{k W})\end{array}$ & $\begin{array}{c}\text { Exergia da } \\
\text { Saída }(\mathbf{k W})\end{array}$ & $\begin{array}{c}\text { Perda de Exergia } \\
(\mathbf{k W})\end{array}$ & $\begin{array}{c}\text { \% de perda } \\
\text { total }\end{array}$ \\
\hline PRETREAT & 497,981 & 127,194 & 370,787 & 74,45 \\
FLASH & 127,194 & 81,643 & 45,551 & 35,81 \\
MIXER & 46,737 & 24,826 & 21,911 & 46,87 \\
HYDROL & 24,826 & 13,624 & 11,202 & 45,11 \\
CENTRIF & 13,624 & 13,624 & 0,000 & 0,00 \\
\hline & TOTAL & 449,451 &
\end{tabular}

A análise exergética apresenta que a perda total de exergia do processo avaliado é de aproximadamente $450 \mathrm{~kW}$. O reator de pré-tratamento foi responsável por aproximadamente $74,45 \%$ de irreversibilidade gerada no balanço exergético do equipamento, sendo a unidade que maior apresentou irreversibilidades no processo estudado. Esse fator ocorre devido às 


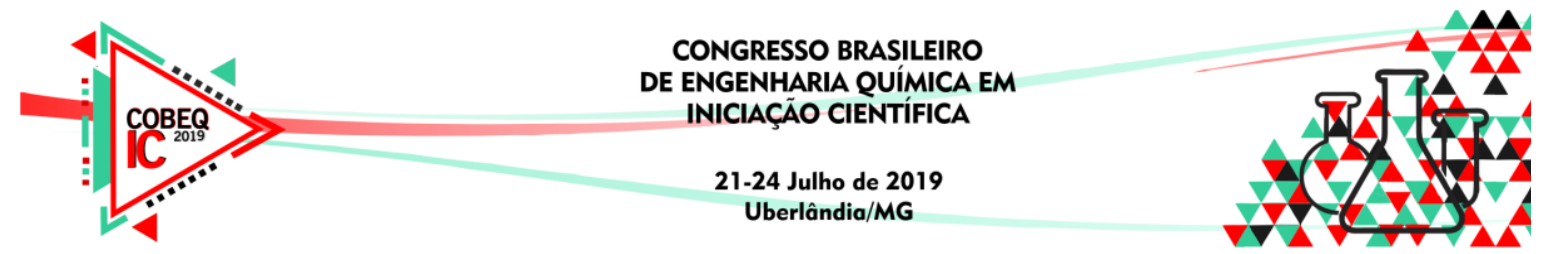

condições da pressão e temperatura de operação do reator, pois a diferença de temperatura é a força motriz da transferência de calor, o que resulta as perdas de exergia serem maiores quando a diferença de temperatura for maior.

A temperatura é um parâmetro de alta influência no processo de pré-tratamento para obter conversões adequadas de etanol na etapa de fermentação. Nesse âmbito, para investigar os principais efeitos de perdas exergéticas na performance do reator foi desempenhada uma análise de sensibilidade do reator de pré-tratamento a diferentes temperaturas de operação, descritas por Jung e Kim (2015), diante o processo ácido diluído. A Figura 3 mostra a análise de sensibilidade das perdas exergéticas do reator no intervalo de $150{ }^{\circ} \mathrm{C}$ a $215^{\circ} \mathrm{C}$.

Figura 3: Análise de sensibilidade da temperatura do reator de pré-tratamento.

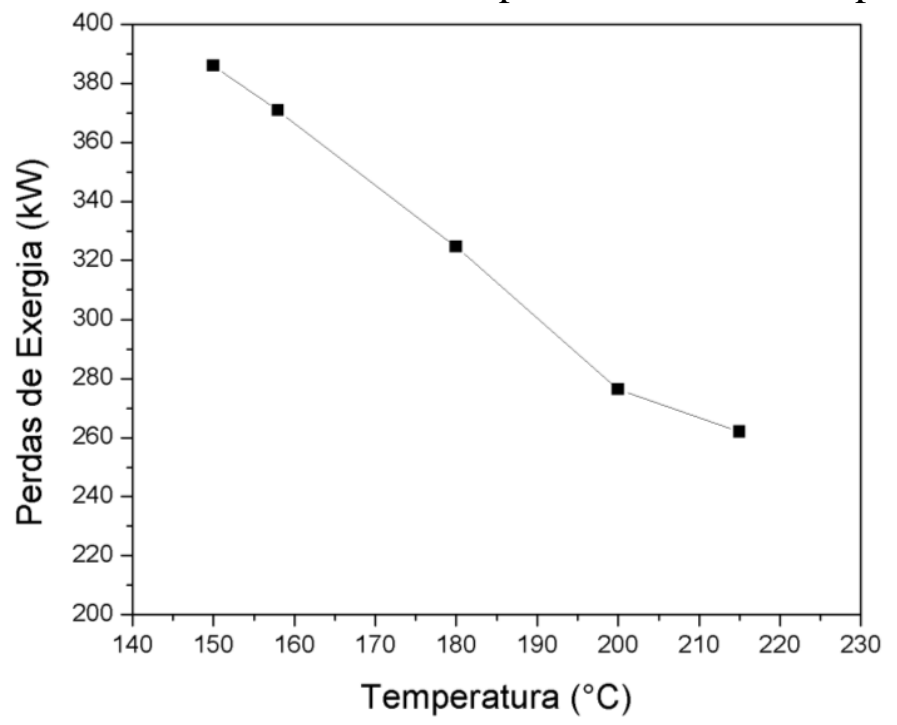

Analisa-se na Figura 3 que o crescimento da temperatura, contribui na diminuição da perda de exergia do reator, devido as condições endotérmicas das reações, as quais proporcionam energia que é consumida pelo próprio sistema. Em síntese, a redução da exergia destruída no reator de pré-tratamento da temperatura de $150{ }^{\circ} \mathrm{C}$ à $215^{\circ} \mathrm{C}$ foi de $32,12 \%$.

\section{CONCLUSÃO}

No presente trabalho, a simulação e avaliação exergética do processo de pré-tratamento ácido diluído da palha de milho seguido por hidrólise enzimática permitiu analisar o desempenho do processo e a influência da temperatura na etapa de pré-tratamento. Os resultados obtidos indicam que a perda de exergia total foi em média de $450 \mathrm{~kW}$, o qual o reator de pré-tratamento apresenta-se como o equipamento de maior perda exergética, além do maior potencial de otimização, devido a sua flexibilidade nas temperaturas de operação. Portanto, o uso do simulador Aspen Plus ${ }^{\circledR}$ apresentou-se como ferramenta de análise de processos químicos extramemente valiosa para identificar a viabilidade do processo e melhorar sua performance energética.

\section{REFERÊNCIAS}




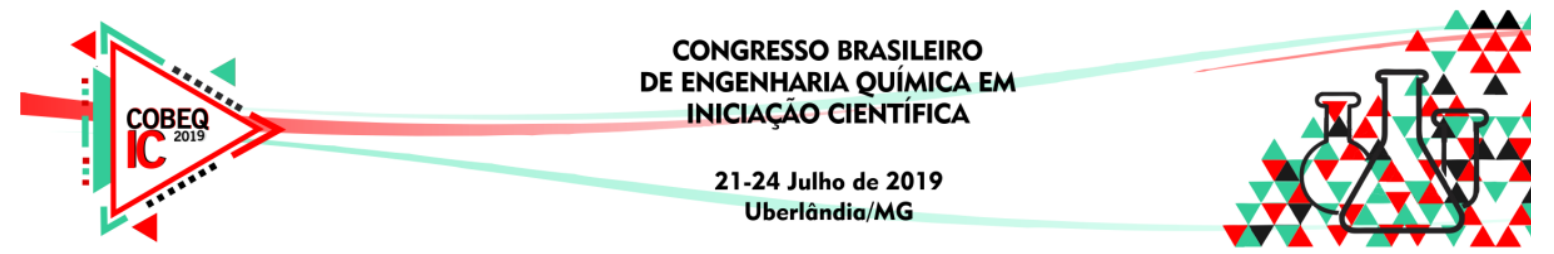

ADITIYA, H.B; MAHLIA, T.M.I; CHONG, W.T. Second generation bioethanol production: A critical review. Renewable and Sustainable Energy Reviews, Malaysia, v. 66, p.631653, 2016.

ASPEN TECH, Aspen Plus Users Manual v.7.2, 2011.

CONDE-MEJÍA, Carolina; JIMÉNEZ-GUTIÉRREZ, Arturo; EL-HALWAGI, Mahmoud. A comparison of pretreatment methods for bioethanol production from lignocellulosic materials. Process Safety and Environmental Protection, v. 90, n. 3, p.189-202, 2012.

HUMBIRD, D. et al. Process Design and Economics for Biochemical Conversion of Lignocellulosic Biomass to Ethanol: Dilute-Acid Pretreatment and Enzymatic Hydrolysis of Corn Stover, Technical Report NREL/TP-5100-47764), National Renewable Energy Laboratory, 2011.

JUNG, Young Hoon; KIM, Kyoung Heon. Acidic Pretreatment. Pretreatment of Biomass. Pretreatment of Biomass: Processes and Technologies, p.27-50, 2015.

LUYBEN, W. L. Process Modelling, Simulation, and Control for Chemical Engineers. 2 ed. Nova Iorque: Editora McGraw Hill, 1996.

OJEDA, Karina; SÁNCHEZ, Eduardo; KAFAROV, Viatcheslav. Sustainable ethanol production from lignocellulosic biomass - Application of exergy analysis. Energy, v. 36, n. 4, p.2119-2128, 2011.

SZARG, Jan. International Progress in Second Law Analysis. Pergamon Press, v. 5, p. 709$718,1980$.

VUČKOVIĆ, Goran D. et al. Advanced exergy analysis and exergoeconomic performance evaluation of thermal processes in an existing industrial plant. Energy Conversion and Management, v. 85, p.655-662, 2014.

WOOLEY RJ, PUTSCHE V: Development of an ASPEN PLUS Physical Property Database for Biofuels Components. Golden, CO: NREL; 1996. 